Investigación Descriptiva, Correlacional o Cualitativa

Pensar en Movimiento:

Revista de Ciencias del Ejercicio y la Salud

ISSN 1659-4436

Vol. 14, No.1, pp. 1-16

Cierre al 30 de junio, 2016

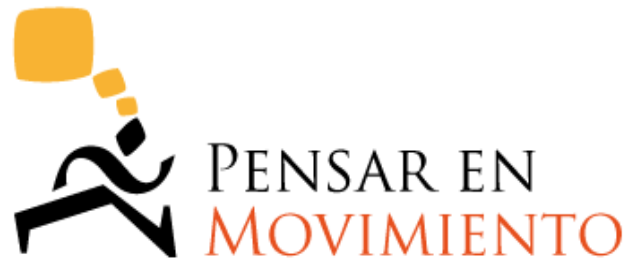

REVISTA DE CIENCIAS DEL EJERCICIO Y LA SALUd

\title{
COMPOSICIÓN CORPORAL, PERFIL LIPÍDICO Y APTITUD FÍSICA EN MUJERES ADOLESCENTES EN MÉXICO
}

\section{BODY COMPOSITION, LIPIDS BLOOD PROFILE AND PHYSICAL FITNESS IN MEXICAN TEENAGE GIRLS}

José Aldo Hernández-Murúa, Ph.D. ${ }^{1(B, C, D, E)}$, Baldomero Llamas-Hernández, Ph.D. ${ }^{1(A, B, C, E)}$, Ciria Margarita Salazar, Ph.D. ${ }^{2(A, B, D, E)}$, Julio Alejandro Gómez-Figueroa, Ph.D. ${ }^{3(A, B, D, E)}$, Cesar Augusto Acuña-Cordova, EMD ${ }^{1(A, B, C, E)}$, José Antonio De Paz-Fernández, Ph.D. ${ }^{4(A, B, D, E)}$

aldohdez80@hotmail.com

${ }^{1}$ Universidad Autónoma de Sinaloa, Sinaloa, México. ²Universidad de Colima, Colima, México.

${ }^{3}$ Universidad de Veracruz, Veracruz, México. ${ }^{4}$ Universidad de León, León, España.

Envío original: 03/03/2016. Reenviado: 20/05/2016, 27/05/2016. Aceptado: 20/06/2016.

Publicado: 24/06/2016

DOI: http://dx.doi.org/10.15517/pensarmov.v14i1.23459

\section{RESUMEN}

Hernández-Murúa, J.A., Llamas-Hernández, B., Salazar, C. M., Gómez-Figueroa, J.A., AcuñaCordova, C.A., De Paz-Fernández, J.A. (2016). Composición corporal, perfil lipídico y aptitud física en mujeres adolescentes en México. Pensar en Movimiento: Revista de Ciencias del Ejercicio y la Salud, 14(1), 1-16. El objetivo de esta investigación fue determinar la composición corporal, el perfil de lípidos sanguíneos y aptitud física en mujeres adolescentes con y sin sobrepeso, y correlacionar cada variable con las demás. Se consideró un diseño transversal. Participaron 38 mujeres adolescentes con edades entre 14 y 19 años. Clasificadas como normopeso $(n=21)$ o sobrepeso $(n=17)$ según la gráfica de la Organización Mundial de la Salud mediante el IMC $\left(\mathrm{kg} / \mathrm{m}^{2}\right)$ en función del puntaje $z$ para edades de 5 a 19 años (puntaje $z: 0 \mathrm{a}+1 D E=$ normopeso, $+1 \mathrm{a}+2 D E=$ sobrepeso). Se realizaron mediciones de composición corporal, perfil de lípidos sanguíneos (colesterol total, triglicéridos, cHDL, cLDL) y aptitud física $\left(\mathrm{VO}_{2 p i c o}\right.$ y $\left.1 \mathrm{RM}\right)$. Los resultados muestran que las adolescentes con normopeso fueron significativamente $(p<0.05)$ más bajas $(M \pm D E)$ en todas las variables de composición corporal que las mujeres adolescentes con sobrepeso. No se encontraron diferencias significativas en el $\mathrm{VO}_{2 p i c o}, 1 \mathrm{RM}$ ni en el perfil de lípidos sanguíneos. En conclusión, las mujeres adolescentes con sobrepeso presentan mayores niveles de composición y grasa corporal pero estos niveles superiores no parecen afectar los niveles de lípidos sanguíneos. Se encontró una 
asociación entre los niveles de aptitud física $\left(\mathrm{VO}_{2 p i c o}\right)$ y la composición corporal (porcentaje de grasa, pliegues cutáneos). Se sugieren otros estudios para buscar la predicción entre los niveles de $\mathrm{VO}_{2 p i c o}$ y grasa corporal.

Palabras claves: porcentaje de grasa corporal; consumo de oxígeno; fuerza muscular; lípidos sanguíneos; sobrepeso.

\section{ABSTRACT}

Hernández-Murúa, J.A., Llamas-Hernández, B., Salazar, C. M., Gómez-Figueroa, J.A., AcuñaCordova, C.A., De Paz-Fernández, J.A. (2016). Body Composition, Lipid Profile and Physical Fitness in Mexican Teenage Girls. Pensar en Movimiento: Revista de Ciencias del Ejercicio y la Salud, 14(1), 1-16. The goal of this research was to measure and correlate body composition, lipid profile and physical fitness in Mexican normal weight and overweight teenage girls by using a cross-sectional study design. The study included 38 adolescent girls (age 14-19) classified as normal weight $(n=17)$ and overweight $(n=21)$, based on the World Health Organization's standards (WHO) with a BMI $\left(\mathrm{kg} / \mathrm{m}^{2}\right)$ depending on the $z$-score; girls and women aged $5-19$ years (z-score: 0 to $+1 S D=$ normal weight, +1 to $+2 S D=$ overweight). Body composition, lipid profile (total cholesterol, triacylglycerol, high-density lipoprotein cholesterol HDL-C, and low-density lipoprotein cholesterol LDL-C), and physical fitness $\left(\mathrm{VO}_{2 \text { peak }}\right.$ and $\left.1 \mathrm{RM}\right)$ were measured. The main findings indicated that body composition variables in normal weight adolescents were significantly lower $(p<0.05, M \pm S D)$ than in overweight adolescent girls. No significant differences were observed in $\mathrm{VO}_{2 \text { peak, }} 1 \mathrm{RM}$ or lipid profile. In conclusion, normal weight teenage girls had a higher body fat composition than overweight teenage girls, but this did not seem to affect lipid levels. However, an association between fitness level $\left(\mathrm{VO}_{2 p e a k}\right)$ and body composition (body fat percent, skinfolds) was found. More studies will be needed to determine the extent to which $\mathrm{VO}_{2}$ levels in adolescent women are associated with their body fat levels.

\section{English translation by Jeannette Soto Segura}

Keywords: Percent body fat; oxygen consumption; muscular strength; blood lipids; overweight.

El sobrepeso y la obesidad son un problema de salud pública en la sociedad del siglo $X X I$. Los niveles de sobrepeso y obesidad son factores de riesgo para enfermedades crónicas como el cáncer, diabetes, hipertensión y cardiopatías isquémicas. Otros dos factores de riesgo asociados a estas enfermedades son altos niveles de lípidos sanguíneos y la inactividad física (Rosillo et al., 2005).

Existen muchos factores que intervienen en el proceso de una enfermedad, no obstante, la composición corporal, el perfil de lípidos sanguíneos y los niveles de condición física en los últimos años han sido muy investigados por ser muy determinantes en la relación saludenfermedad de la población adulta en México y cada vez es más importante estudiarlo en etapas más jóvenes como es la adolescencia (Cervera et al., 2010; Barquera et al., 2013).

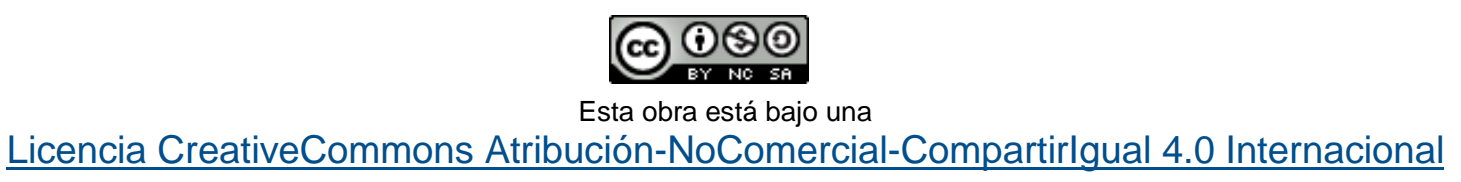


Asimismo, se encuentran varios estudios acerca del sobrepeso y obesidad en personas adultas (Stensvold et al., 2010; Marsh et al., 2013; Tibana et al., 2014; Bruseghini et al., 2015), pero poco se ha enfatizado en México en el estudio específico del sobrepeso en mujeres adolescentes sobre diferentes variables de composición corporal, el perfil de lípidos sanguíneos y su relación con la capacidad aeróbica y muscular.

Hace algunos años, estudios del Instituto Nacional de Salud Pública en México (INSP, 2012) y la Organización para la Cooperación y el Desarrollo Económico (OCDE [por sus siglas en inglés], 2014), han mostrado evidencias que la prevalencia de sobrepeso y obesidad en niños y adolescentes de ambos sexos han incrementado de manera alarmante. Los esfuerzos han sido altos por tratar de describir y dar a conocer los múltiples efectos nocivos del sobrepeso y la obesidad sobre los organismos infantiles y adolescentes.

En 2007 se describió la epidemiología del sobrepeso y la obesidad y su asociación con alteraciones de algunos componentes del síndrome metabólico en una muestra del año 1997, donde los participantes tenían un rango de edad de 10 a 19 años. Tomando los datos de la Encuesta Nacional de Salud (Valdespino et al., 2003), donde el $50.5 \%$ eran mujeres y el 49.5 $\%$ hombres, se encontró que el $14.8 \%$ presentaron sobrepeso, $6.7 \%$ obesidad y el $37.5 \%$ antecedentes familiares de diabetes mellitus tipo 2. Este estudio mostró evidencias de una fuerte asociación entre los niveles de sobrepeso y obesidad con las anormalidades de algunos componentes del síndrome metabólico (Villalpando et al., 2007). En otro estudio llevado a cabo en México se realizó una comparación transversal entre 240 jóvenes obesos y no obesos de 10 a 19 años, para determinar la prevalencia y estimar el riesgo de padecer alguna dislipidemia o hiperinsulinemia, así como evaluar la asociación de medidas antropométricas y lípidos con la insulina. Los resultados mostraron evidencias de la presencia de hiperlipidemia en el $56 \%$ así como un $50 \%$ de hiperinsulinemia de los jóvenes con obesidad; en relación con los niveles de insulina se identificó una correlación significativa con el índice de masa corporal (MarcosDaccarett et al., 2007).

En el 2001 en Singapur se realizó un estudio para determinar los niveles del perfil de lípidos sanguíneos (triglicéridos, cHDL, insulina y glucosa) en niños obesos y no obesos, los resultados mostraron evidencias que los niños con obesidad presentaban niveles más altos en la circunferencia de la cintura, mayores niveles de triglicéridos, insulina, así como menores niveles de consumo de oxígeno pico (valores de $\mathrm{VO}_{2 \text { pico }}$ expresados en $\mathrm{ml} \cdot \mathrm{kg} \cdot \mathrm{min}^{-1}$ ) (Stensel et al., 2001). Otros factores como la inactividad física y el sedentarismo han sido identificados como factores de riesgo asociados con una mayor incidencia de enfermedades crónicas no transmisibles (García-Artero et al., 2007). Entre las cualidades asociadas para incrementar la actividad física están los ejercicios de carácter aeróbico y fuerza muscular. Estas cualidades han sido asociadas a que contribuyen a disminuir los niveles de composición corporal, los altos índices de lípidos en la sangre e incrementan la condición física de las personas (Hanai et al., 1999; Norman et al., 2005; Boer et al., 2013) para estabilizar y mejorar su estado salud. En México no se han encontrado evidencias en un único estudio que hayan comparado diferentes

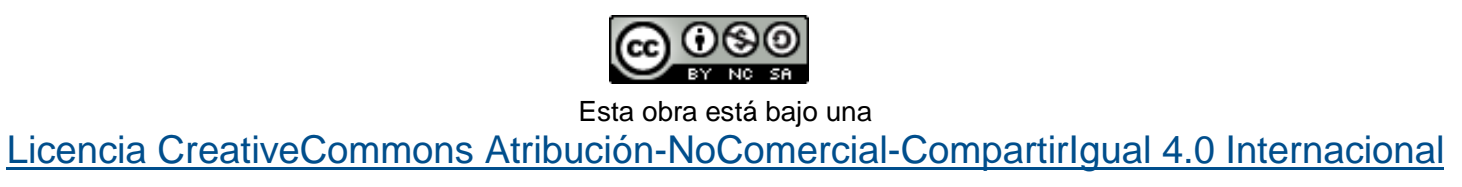


variables de composición corporal, el perfil de lípidos sanguíneos y la aptitud aeróbica y muscular en mujeres adolescentes con sobrepeso versus peso normal ni la asociación entre ellas. Nuestro objetivo fue comparar diferentes variables de composición corporal (IMC, \% de grasa, la circunferencia cintura, la ICC), los niveles de perfil de lípidos sanguíneos (colesterol total, triglicéridos, c-HDL, c-LDL, TC/HDL, lípidos totales), y la capacidad aeróbica y muscular en mujeres adolescentes con y sin sobrepeso.

\section{METODOLOGÍA}

Participantes. Este estudio se realizó con un grupo de 21 con peso normal y el segundo grupo de 17 con sobrepeso, ambos formados por mujeres adolescentes mexicanas con edades comprendidas entre 14 y 19 años (Tabla 1). Antes de iniciar el estudio se obtuvo por escrito el consentimiento informado de las adolescentes y de sus padres. La Junta de Revisión Institucional y el Comité de Ética de la Escuela Superior de Educación Física aprobaron el estudio antes de la recolección de los datos. Todas las participantes y sus padres fueron informados de los propósitos y posibles riesgos involucrados en el estudio, antes de obtener sus informes de consentimiento por escrito como participantes. Las adolescentes se clasificaron considerando el índice de masa corporal (IMC) por edad en normopeso y sobrepeso; según el gráfico y la clasificación de la Organización Mundial de la Salud (WHO [por sus siglas en inglés], 2007) para infantes y adolescentes de 5 a 19 años en función de los puntajes estandarizados $z$ y el sexo, datos obtenidos para cada adolescente en donde se comparó su IMC con la referencia de la OMS de su propia edad, clasificadas en dos grupos; normopeso $[(0 \mathrm{a}+1$ desviación estándar $(D E)]$ y sobrepeso $(+1 \mathrm{y}+2 D E)$. El estado puberal no fue determinado, pero no se encontraron diferencias significativas entre los grupos con respecto a la edad y altura (Tabla 1).

Tabla 1

Características físicas de mujeres adolescentes del estudio

\begin{tabular}{|c|c|c|c|}
\hline & $\begin{array}{l}\text { Normopeso } \\
(n=21)\end{array}$ & $\begin{array}{c}\text { Sobrepeso } \\
(n=17)\end{array}$ & Significancia \\
\hline Edad (años) & $\begin{array}{r}16.8 \pm 0.9 \\
(15-19)\end{array}$ & $\begin{array}{r}16.4 \pm 1.3 \\
(14-19)\end{array}$ & .3717 \\
\hline Altura (m) & $\begin{array}{c}1.6 \pm 0.1 \\
(1.5-1.7)\end{array}$ & $\begin{array}{c}1.6 \pm 0.1 \\
(1.3-1.7)\end{array}$ & .5882 \\
\hline Peso (kg) & $\begin{array}{l}53.8 \pm 4.9 \\
(45.7-64.8)\end{array}$ & $\begin{array}{r}68.3 \pm 8.5 * \\
(48.5-81.8)\end{array}$ & .0001 \\
\hline Nota: Valores s & entan $M \pm D E$ (val & $\begin{array}{l}- \text { máximo); }{ }^{*} p<0.0 \\
-4-\end{array}$ & nte: Elaboracić \\
\hline
\end{tabular}


Composición corporal. Para las mediciones de composición corporal se citó a las participantes para que llegaran con ropa ligera, calcetines, shorts y blusa deportiva. La altura fue medida con una precisión de $0.1 \mathrm{~cm}$ con un altímetro (Asimed, modelo MB 201 Plus) en centímetros $(\mathrm{cm})$. El peso corporal se determinó con una precisión de $0.1 \mathrm{~kg}$ con una báscula digital (Tanita, modelo BF-680W). La circunferencia cintura y cadera se midió en centímetros con una cinta métrica con retracción automática (Seca, modelo 201). La medición de la cintura se realizó con la joven de pie en una posición cómoda, se colocó la cinta en la parte más estrecha entre el borde costal inferior y la cresta iliaca en el plano horizontal después de una espiración normal. La cadera se midió a nivel de la mayor protuberancia glútea, de tal manera que la parte anterior coincidiera con la sínfisis púbica. El índice de cintura cadera (ICC) se calculó considerando las circunferencias de cintura $(\mathrm{cm}) /$ cadera $(\mathrm{cm})$.

El índice de masa corporal (IMC) fue calculado de la siguiente forma: peso $(\mathrm{kg}) /$ altura$^{2}\left(\mathrm{~m}^{2}\right)$. Se utilizó un calibrador de pliegues cutáneos (Holtein®, Dyfed, UK) para determinar los pliegues cutáneos de las regiones del bíceps, tríceps, subescapular, abdominal, suprailíaco, muslo y pantorrilla con una precisión de $0.1 \mathrm{~cm}$. Después de la recolección de los datos el porcentaje de grasa corporal (\% grasa corporal) fue determinado usando la ecuación de Slaugther (Dezenberg et al., 1999). Además, se calcularon dos indicadores de adiposidad con la suma de cinco (Vaeyens et al., 2006) y cuatro (Lazaar et al., 2007) pliegues cutáneos, previamente utilizados en otro estudio en adolescentes. Las evaluaciones antropométricas del estudio fueron realizadas de acuerdo con estudios previamente publicados (FernándezGonzalo et al., 2010). Las variables dependientes seleccionadas para este estudio para definir la composición corporal fueron el IMC, el \% grasa corporal, la circunferencia cintura (CC) y el ICC, todas comúnmente usadas en diferentes estudios para la valoración de la composición corporal.

\section{Aptitud física $\left(\mathrm{VO}_{2}\right.$ pico y $\left.1 \mathrm{RM}\right)$.}

$\mathrm{VO}_{2}$ pico. Para la evaluación de la aptitud física aeróbica se midió el consumo de oxígeno pico $\left(\mathrm{VO}_{2 p i c o}\right)$ como prueba estándar de capacidad de ejercicio aeróbico y aptitud cardiorrespiratoria previamente utilizado en otro estudio (Stenselet al., 2001). Tras la familiarización de las participantes se determinó el $\mathrm{VO}_{2 p i c o}$ individual usando una banda sin fin (Scifit@DC4000, UK). Se llevó a cabo un protocolo incremental con una velocidad inicial de 4.0 $\mathrm{km} \cdot \mathrm{h}^{-1}$ e incrementos de $1.0 \mathrm{~km} \cdot \mathrm{h}^{-1}$ cada minuto hasta el agotamiento máximo alcanzado por cada participante (Fernández-Gonzalo et al., 2010). Durante el test se realizó el análisis $\mathrm{VO}_{2 p i c o}$ respiración a respiración (Cosmed®Fitmate, Roma, Italia). El gas analizado y la medición de aire fue calibrado antes de cada prueba según las instrucciones del fabricante y fue medido el consumo de $\mathrm{VO}_{2 p i c o}$ continuamente en cada inspiración y expiración de aire. Para el análisis de los datos se consideró el $\mathrm{VO}_{2 p i c o}$ en dos unidades $\left(\mathrm{ml} \cdot \mathrm{kg} \cdot \mathrm{min}^{-1}\right.$ y L·min-1) (Sassenet al., 2010).

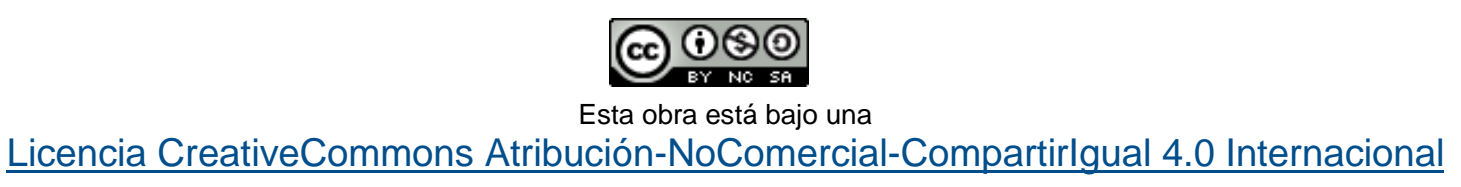


Fuerza máxima dinámica muscular (1RM). Para la evaluación de la aptitud muscular se midió la fuerza dinámica máxima muscular o también conocida como 1RM en los miembros inferiores en el ejercicio de press de pierna con una inclinación a $45^{\circ}$ (GervaßSport, España). Tras la familiarización de las participantes se determinó 1RM; el protocolo consistió en un calentamiento general de 5 minutos en cicloergómetro, seguido por un calentamiento específico de 10 repeticiones con un peso auto-seleccionado de entre un 30-50 \% estimado de 1RM. Para el 1RM en press de pierna se tenía que ejecutar una contracción en fase concéntrica lo más rápido posible partiendo de una posición de $90^{\circ}$ de flexión de articulación de rodillas hacia una extensión de $180^{\circ}$. Si el intento se completaba correctamente se incrementaba la carga entre 20 y 40 kilogramos hasta alcanzar 1RM; Si el intento no se completaba correctamente, se retiraba el peso y se le daba otra oportunidad. Un mínimo de 3 minutos de recuperación se daba entre cada intento. Este procedimiento se continuó hasta completar 1RM, típicamente alcanzando el máximo valor entre 4-6 intentos. Para el análisis de los datos se consideró la fuerza máxima relativa: los kilogramos de fuerza aplicada $(\mathrm{kg})$ divididos por el peso corporal en kilogramos $(\mathrm{kg})$, y expresados en múltiplos del peso corporal (PC). Esta metodología ha sido publicada previamente en otros protocolos (Hollander et al., 2007; Halet et al., 2009).

Perfil de lípidos sanguíneos. Para el análisis del perfil de lípidos sanguíneos, las participantes fueron citadas por la mañana a un laboratorio clínico de bioquímica comercial considerando un ayuno de entre 10-12 h y las normas de bioseguridad. Se les pidió a las participantes que no realizaran ejercicio extenuante en las $48 \mathrm{~h}$ previas al análisis. Se les extrajo una muestra de sangre venosa $(20 \mathrm{ml})$ y se colocó en tubos sin aditivos, la cual se centrifugó a $2000 \mathrm{rpm}$ durante $10 \mathrm{~min}$ a una temperatura de $4^{\circ} \mathrm{C}$, para la posterior extracción de suero y plasma, respectivamente, para la realización del análisis bioquímico. Se realizaron determinaciones del perfil de lípidos sanguíneos de colesterol total (CT), triglicéridos (TG), lipoproteínas de alta densidad (cHDL) y se calculó el índice de riesgo aterogénico (CT/cHDL). Se utilizaron métodos enzimáticos colorimétricos utilizando kits comerciales Bayer con un analizador RA-50 (SystemDiagnostic, Dublin, Irlanda). Para el cálculo de las lipoproteínas de baja densidad (CLDL) se utilizó la fórmula de Friedewald ajustada por las concentraciones séricas de triglicéridos (Friedewald et al.,1972; Nakanishi et al.,2000).

Análisis Estadístico. El tratamiento de los datos se realizó mediante el paquete Statistical Package for Social Sciences (SPSS versión 22.0). La estadística descriptiva de los resultados se muestra mediante el valor de la media $(M) \pm$ la desviación estándar $(D E)$. La normalidad de la distribución de las variables fue examinada con las pruebas de la normalidad de Kolmogorov-Smirnov. La diferencia entre mujeres adolescentes con normopeso y sobrepeso fue evaluada usando la prueba t para muestras independientes. Para la correlación se aplicó la prueba de Pearson. El nivel de significación fue establecido para una $p<0.05$.

$-6-$

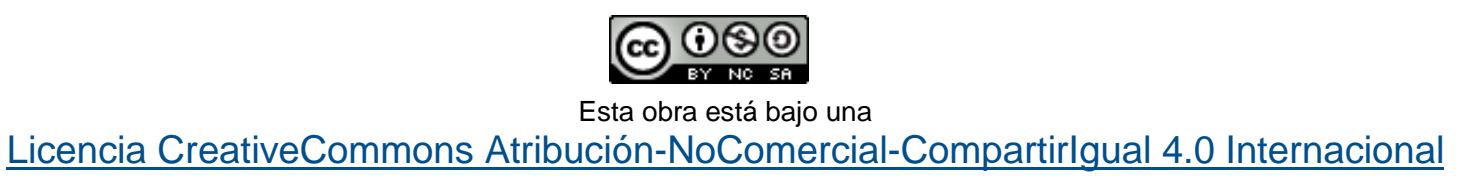


Tabla 2

Composición corporal y aptitud física en mujeres adolescentes del estudio

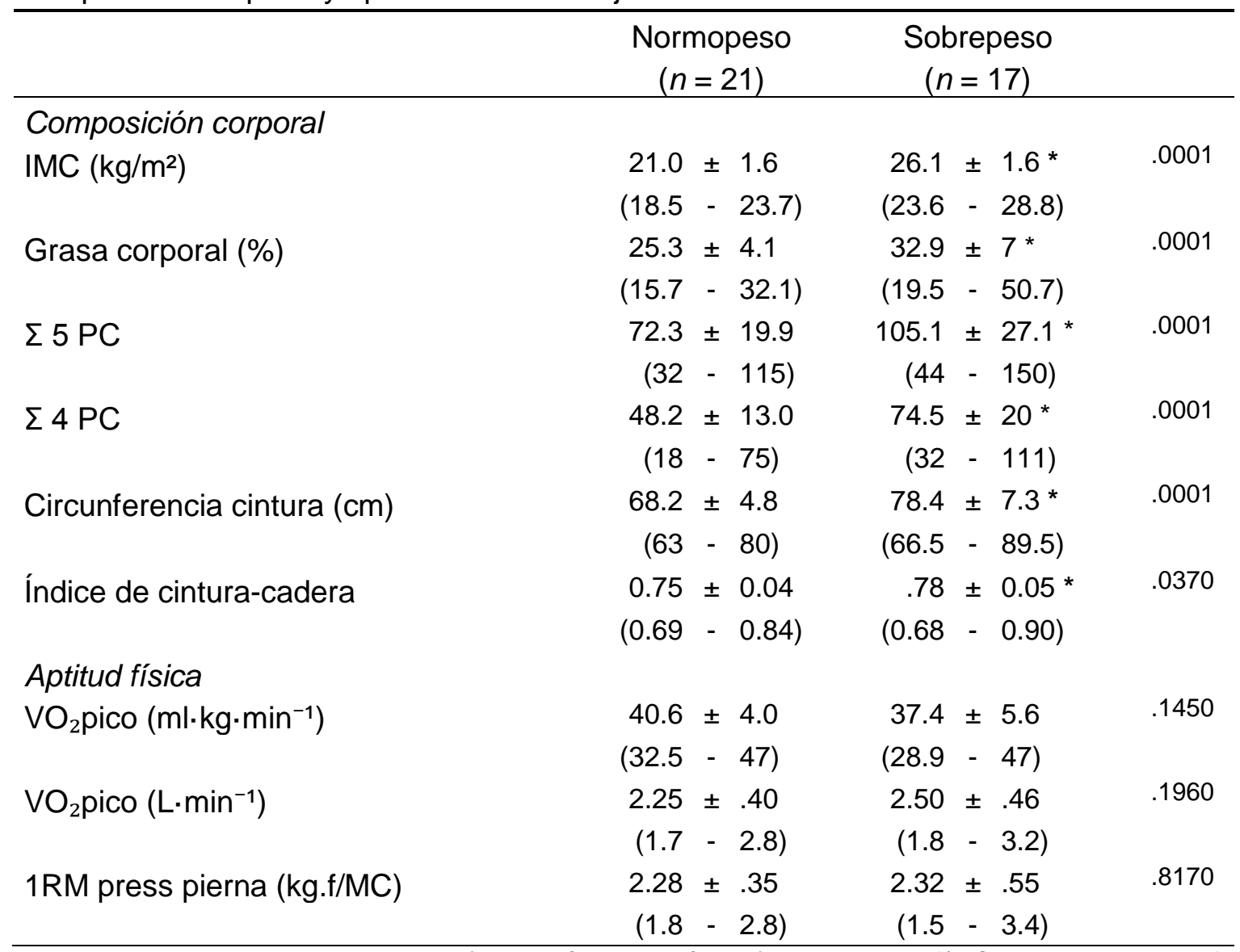

Nota: Valores se presentan $M \pm D E$ (valor mínimo - máximo); ${ }^{*} p<0.05 . \mathrm{kg} . \mathrm{f} / \mathrm{MC}=$ Kilogramos de fuerza aplicada/masa corporal en kilogramos. Fuente: Elaboración propia

\section{RESULTADOS}

Composición corporal. En la edad y altura no se encontraron diferencias significativas entre los grupos, no obstante, las adolescentes con sobrepeso presentaron significativamente más peso que las de normopeso, como era de esperar (ver Tabla 1). Los resultados de composición corporal muestran que las adolescentes con sobrepeso en todas las variables presentaron valores más altos significativamente $(p<0.05)$, de tal manera que se manifiesta

\section{(c) (1) (2)}

Esta obra está bajo una Licencia CreativeCommons Atribución-NoComercial-Compartirlgual 4.0 Internacional 
completamente el debido exceso de composición corporal y adiposidad en comparación a las de normopeso (Tabla 2).

Aptitud física ( $\mathrm{VO}_{2}$ pico y 1RM). En la Tabla 2 también se muestran los resultados de los valores de $\mathrm{VO}_{2 \text { pico }}$ tanto en valores relativos $\left(\mathrm{ml} \cdot \mathrm{kg} \cdot \mathrm{min}^{-1}\right)$ como absolutos $\left(\mathrm{L} \cdot \mathrm{min}^{-1}\right)$. Se ha podido constatar que no existen diferencias significativas en el $\mathrm{VO}_{2 \text { pico }}$ entre las adolescentes con y sin sobrepeso. De la misma manera, en los valores de la aptitud física muscular dinámica (1RM) en piernas no se encontró diferencia entre ambos grupos.

Perfil de lípidos sanguíneos. En la Tabla 3 se muestran los metabolitos (perfil de lípidos) sanguíneos examinados en las participantes de este estudio (normopeso vs. sobrepeso). Se pudo observar que de todas las variables estudiadas: el colesterol total (CT), los triglicéridos (TG), las lipoproteínas de alta (cHDL) y baja (cLDL) densidad y el índice aterogénico (CT/cHDL), ninguna presentó diferencias significativas entre ambos grupos de este estudio. Se observaron correlaciones entre el $\mathrm{VO}_{2 p i c o}$ y varios parámetros de composición corporal (el porcentaje de grasa corporal y la suma de 4 y 5 pliegues cutáneos se pueden ver en la Tabla 4). No obstante, no se ha podido comprobar evidencias de correlaciones del perfil de lípidos sanguíneos con los parámetros de composición corporal, el $\mathrm{VO}_{2 p i c o}$ y la fuerza muscular. Los resultados medidos de correlación se pueden ver en la Tabla 4.

\section{DISCUSIÓN}

Los principales resultados de este estudio fueron que todas las variables de la composición corporal analizadas (IMC, \% grasa, Adiposidad, CC y ICC) fueron más altas en las mujeres adolescentes con sobrepeso que las de normopeso. Mientras que la aptitud física aeróbica $\left(\mathrm{VO}_{2 p i c o}\right)$, muscular (1 $\mathrm{RM}$ ) y las diferentes variables del perfil de lípidos sanguíneos no mostraron diferencias entre ambos grupos.

$-8-$

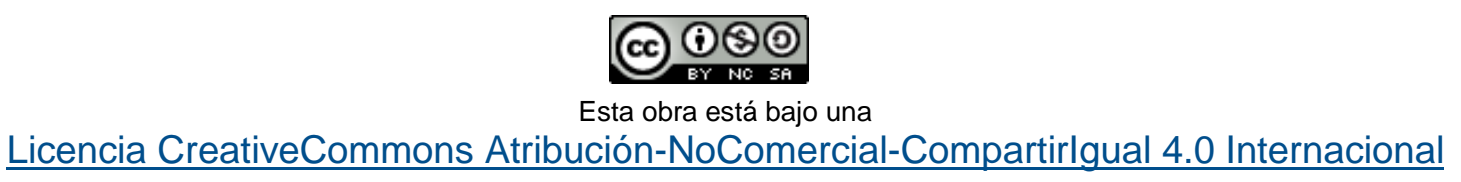




\section{Tabla 3}

Metabolitos sanguíneos en mujeres adolescentes

\begin{tabular}{lrrrr}
\hline & \multicolumn{2}{c}{$\begin{array}{c}\text { Normopeso } \\
(n=21)\end{array}$} & $\begin{array}{c}\text { Sobrepeso } \\
(n=17)\end{array}$ & Significancia \\
\hline CT $(\mathrm{mg} / \mathrm{dl})$ & $149.0 \pm 32.4$ & $144.2 \pm 21.9$ & .982 \\
TG $(\mathrm{mg} / \mathrm{dl})$ & $60.7 \pm 27.8$ & $73.9 \pm 43.4$ & .438 \\
cHDL $(\mathrm{mg} / \mathrm{dl})$ & $52.2 \pm 8.7$ & $48.0 \pm 9.6$ & .420 \\
CLDL $(\mathrm{mg} / \mathrm{dl})$ & $84.6 \pm 28.0$ & $81.4 \pm 17.2$ & .912 \\
CT/HDL-C & $2.90 \pm .62$ & $3.10 \pm 0.7$ & .407 \\
\hline
\end{tabular}

Nota: Valores se presentan $M \pm D E$ (valor mínimo y máximo). $C T=$ Colesterol total; $T G=$ Triglicéridos; $\mathrm{cHDL}=$ Lipoproteínas de alta densidad; $\mathrm{CLDL}=$ Lipoproteínas de baja densidad; CT/cHDL = Índice de riesgo aterogénico. Fuente: Elaboración propia.

\section{Tabla 4}

Correlación de Pearson $(n=38)$ entre la composición corporal, aptitud física y perfil de lípidos sanguíneos

\begin{tabular}{lcccccc} 
& IMC & $\%$ Grasa & $\Sigma 5$ PC & $\Sigma 4$ PC & CC & ICC \\
\hline IMC & 1 & $.764^{* *}$ & $.709^{* *}$ & $.750^{* *}$ & $.821^{* *}$ & $.428^{* *}$ \\
VO2pico $\left(\mathrm{ml} \cdot \mathrm{kg} \cdot \mathrm{min}^{1}\right)$ & -.333 & $-.624^{* *}$ & $-.615^{* *}$ & $-.613^{* *}$ & -.246 & -.081 \\
CT/cHDL & .265 & .333 & .303 & .322 & .279 & .281 \\
CT & .025 & .309 & .306 & .315 & .141 & .026 \\
TG & .281 & .231 & .229 & .248 & .208 & .150 \\
CHDL & -.238 & -.060 & -.026 & -.047 & -.120 & -.275 \\
CLDL & .040 & .324 & .308 & .321 & .154 & .094 \\
\hline
\end{tabular}

IMC = índice de masa corporal; \% = Porcentaje de grasa corporal; ICC = Índice cintura/cadera; $\Sigma 5$ y 4 $\mathrm{PC}=$ Suma de 5 y 4 pliegues cutáneos respectivamente; $\mathrm{CC}=$ Circunferencia cintura; $\mathrm{CT} / \mathrm{cHDL}=$ índice de riesgo aterogénico; $\mathrm{CT}=$ Colesterol total; $\mathrm{TG}=$ triglicéridos; $\mathrm{cHDL}=$ Lipoproteínas de alta densidad; $\mathrm{cLDL}=$ Lipoproteínas de baja densidad. ${ }^{*}$ r-Pearson diferencia significativa $p<0.05$. Fuente:

Elaboración propia

La gran mayoría de los estudios que han aportado evidencias científicas se centran en analizar el sobrepeso y obesidad en niños y adolescentes, no hacen una comparativa exclusiva entre adolescentes con sobrepeso contra normopeso, como se ha planteado en este estudio en mujeres adolescentes con sobrepeso porque según datos publicados por la OCDE (OCDE, 2014) el $29 \%$ de adolescentes del sexo femenino padecen sobrepeso en México, es decir una

$-9-$

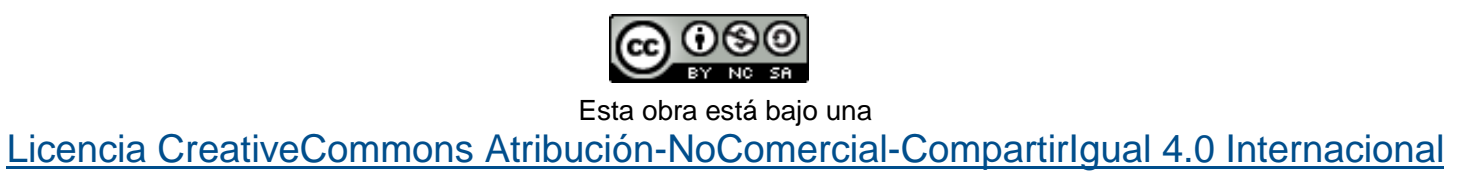


de cada tres adolescentes. Se destaca que en este estudio la capacidad aeróbica $\left(\mathrm{VO}_{2 p i c o}\right)$ ni la fuerza muscular dinámica (1RM) fueron diferentes entre ambos grupos. Al comparar los valores de $\mathrm{VO}_{\text {2pico }}$ (normopeso: $40.6 \mathrm{ml} \cdot \mathrm{kg} \cdot \mathrm{min}^{-1}$; sobrepeso: $37.4 \mathrm{ml} \cdot \mathrm{kg} \cdot \mathrm{min}^{-1}$ ) de este estudio, se observó que fueron muy similares a los publicados por Stensel et al. (2001) que presentaron valores de $41.6 \mathrm{ml} \cdot \mathrm{kg} \cdot \mathrm{min}^{-1}$ (en un grupo de normopeso) y de $33.8 \mathrm{ml} \cdot \mathrm{kg} \cdot \mathrm{min}^{-1}$ (en un grupo de obesidad). A diferencia de esta investigación Stensel et al., sí observaron diferencias entre sus grupos. Al comparar los valores de $1 \mathrm{RM}$ en términos relativos (normopeso: 2.28 PC; sobrepeso: $2.32 \mathrm{PC}$ ), por lo que se observó que es superior al publicado por Hollander et al. (2007) en un grupo diez mujeres jóvenes (con normopeso: 1.62 PC), quizás por la configuración de la máquina de press de piernas. Estos datos indican que el sobrepeso no es un factor determinante sobre la aptitud aeróbica y muscular y por otro lado, estas pueden ser bien desarrolladas similarmente a las mujeres adolescentes con peso normal para futuros programas de mejoría de la salud y calidad de vida de la población.

En la literatura existen una variedad de métodos para la evaluación y clasificación del sobrepeso y obesidad en hombres y mujeres adolescentes (Brandon, 1998; Chan et al., 2009; Cole et al., 2000; Yeung y Hui, 2010; López et al., 2011, Stensel et al., 2001).En este estudio se consideró el IMC como variable de clasificación previamente descrita en población adolescentes (Salazar-Martínez et al., 2006; Abdulrazzaq et al., 2011), porque está bien correlacionada con las condiciones de comorbilidad en niños y adolescentes (Krebs y Jacobson, 2003). Las mujeres adolescentes con sobrepeso presentaron un exceso de peso de $13.4 \mathrm{~kg}$, significativamente diferente a las de normopeso. Sin embargo, aún cuando el grupo con sobrepeso presentó mayor exceso de grasa los resultados son alentadores, ya que indican que el sobrepeso en mujeres adolescentes de este estudio aún no ha afectado ni se han presentado repercusiones adversas sobre los niveles de aptitud física cardiovascular, de fuerza músculo-esquelética, ni en los niveles de lípidos sanguíneos. Contrariamente a este estudio Stensel et al., (2001) realizaron otro, donde comparan el perfil de lípidos sanguíneos entre niños obesos y no obesos con edades promedios de 13 años en el cual encontraron alteraciones en los niveles de triglicéridos y la insulina.

En este estudio se encontró una relación clara entre el $\mathrm{VO}_{2 \text { pico }}$ con el IMC y los niveles de adiposidad con la suma de cuatro y cinco pliegues cutáneos, mientras que con los indicadores CC e ICC no se observó ninguna relación, como se puede analizar en la Tabla 4. Esta correlación encontrada fue negativa, lo que significa que cuanto mayor son los valores de $\mathrm{VO}_{2 \text { ico }}$ mejores índices de IMC y niveles de adiposidad pueden ser encontrados en mujeres adolescentes. Lo que destacaría el impacto del ejercicio para contrarrestar altos grados de sobrepeso y obesidad en adolescentes en México. No obstante, en los resultados de este estudio no se pudo comprobar correlación entre los valores de composición corporal y aptitud física versus los valores del perfil de lípidos sanguíneos. En este aspecto los resultados de este estudio fueron contrarios a lo publicados en otros, puesto que encontraron una asociación entre los niveles de $\mathrm{VO}_{2 \text { pico }}$ y fuerza muscular con los niveles de perfil lipídico-metabólico en 248

$-10-$

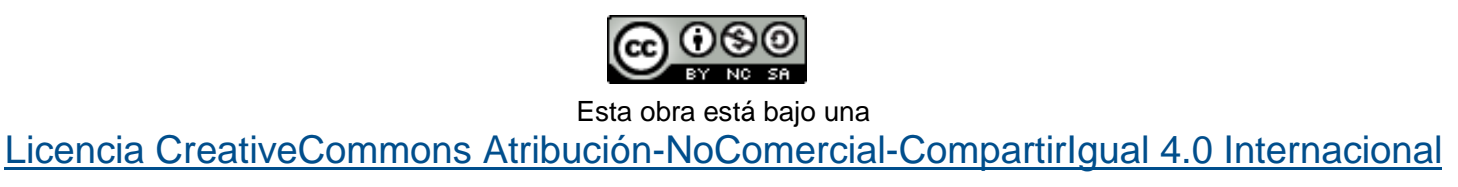


varones y 212 mujeres con peso normal en edades promedios de 15 años (García-Artero et al., 2007). Estos autores, en comparación con los resultados del presente estudio, aplicaron el test de Course-Navette para la valoración del $\mathrm{VO}_{2 p i c o}$ y la fuerza general mediante el promedio de las siguientes tres pruebas físicas de campo: a. test de salto en longitud sin impulso y con pies juntos para evaluar la fuerza explosiva de tren inferior, b. test de dinamometría manual para evaluar la fuerza máxima de prensión manual, c. test de suspensión en barra hasta el agotamiento con flexión de brazos para evaluar la fuerza-resistencia de tren superior; encontraron que una buena capacidad aeróbica en varones adolescentes (varones $=\mathrm{VO}_{2}>49.2$ $\mathrm{ml} \cdot \mathrm{kg} \cdot \mathrm{min}^{-1}$ ) está asociada con un menor índice de riesgo cardiovascular, independientemente del grado de actividad física y fuerza muscular; mientras que en mujeres adolescentes que poseen mayores valores de fuerza presentan un perfil lipídico-metabólico más saludable que aquellas que presentaron valores menores.

Esta diferencia encontrada en relación con este estudio pueden tener una explicación en varias direcciones: primero, la pequeña muestra usada; segundo, los valores de todas las variables del perfil de lípidos sanguíneos están dentro de los parámetros normales en ambos grupos, lo que indica que las adolescentes de ambos grupos de la actual investigación no presentaron riesgos de perfil metabólico o riesgo de enfermedad cardiovascular; tercero, García-Artero et al. (2007) utilizaron diferentes protocolos para la valoración del $\mathrm{VO}_{2 p i c o}$ y fuerza muscular; por último los datos publicados por García-Artero et al. (2007) fueron superiores a los de este estudio.

En conclusión, este estudio contribuye que las mujeres adolescentes con sobrepeso, a pesar de presentar mayores niveles de composición y grasa corporal (en todas las variables estudiadas), esto no pareciera afectar los niveles de lípidos. Se encontró una asociación entre los niveles de aptitud física $\left(\mathrm{VO}_{2 \text { pico }}\right)$ y los porcentajes de grasa corporal (\% grasa, pliegues cutáneos). Se sugieren otros estudios para buscar la predicción entre los niveles de aptitud física y grasa corporal.

\section{REFERENCIAS}

Abdulrazzaq, Y. M., Nagelkerke, N., \& Moussa, M. A. (2011). UAE population reference standard charts for body mass index and skinfold thickness, at ages 0-18 years. International Journal of Food Sciences and Nutrition, 62(7), 692-702. doi: http://dx.doi.org/10.3109/09637486.2011.567978

Barquera, S., Campos-Nonato, I., Hernández-Barrera, L., Pedroza, A., \& Rivera-Dommarco, J. A. (2013). Prevalence of obesity in Mexican adults 2000-2012. Salud Pública de

$-11-$

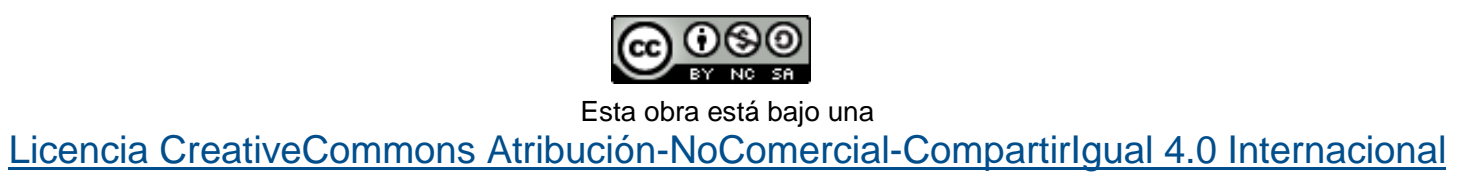


México, 55(2). Recuperado de http://www.scielosp.org/scielo.php?pid=S0036$\underline{36342013000800012 \& \text { script }=\text { sci arttext }}$

Boer, P. H., Meeus, M., Terblanche, E., Rombaut, L., De Wandele, I., Hermans, L., Gysel, T., Ruige, J., \& Calders, P. (2013). The influence of sprint interval training on body composition, physical and metabolic fitness in adolescents and young adults with intellectual disability: a randomized controlled trial. Clinical rehabilitation, 28(3), 221-231. Recuperado

de http://cre.sagepub.com/content/early/2013/08/19/0269215513498609.abstract

Brandon, L. (1998). Comparison of existing skinfold equations for estimating body fat in African American and white women. The American Journal of Clinical Nutrition, 67(6), 11551161. Recuperado de http://ajcn.nutrition.org/content/67/6/1155.short

Bruseghini, P., Calabria, E., Tam, E., Milanese, C., Oliboni, E., Pezzato, A., Pogliaghi, S., Luca, G., Schena, R., \& Capelli, C. (2015). Effects of eight weeks of aerobic interval training and of isoinertial resistance training on risk factors of cardio metabolic diseases and exercise capacity in healthy elderly subjects.Oncotarget,6(19).doi: http://dx.doi.org/10.18632/oncotarget.4031

Chan, DFY., Li, A. M., So, HK., Yin, J., \& Nelson, EAS. (2009). New skinfold-thickness equation for predicting percentage body fat in Chinese obese children. Hong Kong Journal of Pediatr, 14(2), 96-102. Recuperado de URL:http://hkjpaed.org/details.asp?id=694\&show=1234

Cervera, S. B., Campos-Nonato, I., Rojas, R., \& Rivera, J. (2010). Obesidad en México: epidemiología y políticas de salud para su control y prevención. Gaceta Médica de México. Órgano Oficial de la Academia Nacional de Medicina de México, AC, 146, 397407. URL:http://www.medigraphic.com/pdfs/gaceta/gm-2010/gm106g.pdf

Cole T.J., Bellizzi M.C., Flegal K.M., Dietz W.H. (2000). Establishing a standard definition for child overweight and obesity worldwide: international survey. British Medical Journal (320), 1240-1243.doi:http://dx.doi.org/10.1136/bmi.320.7244.1240

Dezenberg, C. V., Nagy, T. R., Gower, B. A., Johnson, R., \& Goran, M. I. (1999). Predicting body composition from anthropometry in pre-adolescent children. International Journal of Obesity, 23(3), 253-259. doi: http://dx.doi.org/10.1038/sj.ijo.0800802 
Fernández-Gonzalo, R., De Souza-Teixeira, F., Bresciani, G., García-López, D., HernándezMurúa, J. A., Jiménez-Jiménez, R., \& De Paz, J. A. (2010). Comparison of technical and physiological characteristics of prepubescent soccer players of different ages. The Journal of Strength \& Conditioning Research, 24(7), 17901798.doi:http://dx.doi.org/10.1519/JSC.0b013e3181def871

Friedewald, W.T., Levy, R.I., Fredrickson, D.S. (1972). Estimation of the concentration of lowdensity lipoprotein cholesterol in serum, without use of the preparative ultracentrifuge. Clinical Chemistry, 18(6), 499-502. Recuperado de http://www.clinchem.org/content/18/6/499.short

García-Artero, E., Ortega, F. B., Ruiz, J. R., Mesa, J. L., Delgado, M., González-Gross, M., García-Fuentes, M., Vicente-Rodríguez, G., Gutiérrez, A \& Castillo, M. J. (2007). El perfil lipídico-metabólico en los adolescentes está más influido por la condición física que por la actividad física (estudio AVENA). Revista española de Cardiología, 60(6), 581588.doi:http://dx.doi.org/10.1157/13107114

Halet, K. A., Mayhew, J. L., Murphy, C., \& Fanthorpe, J. (2009). Relationship of 1 repetition maximum lat-pull to pull-up and lat-pull repetitions in elite collegiate women swimmers. The Journal of Strength \& Conditioning Research, 23(5), 1496-1502. doi: http://dx.doi.org/10.1519/JSC.0b013e3181b338ec

Hanai, T., Takada, H., Nagashima, M., \& Iwata, T. K. (1999). Effects of exercise for 1 month on serum lipids in adolescent females. Pediatrics international, 41(3), 253-259. Recuperado de http://onlinelibrary.wiley.com/doi/10.1046/j.1442-200x.1999.01064.x/full

Hollander, D. B., Kraemer, R. R., Kilpatrick, M. W., Ramadan, Z. G., Reeves, G. V., Francois, M., Herbet, E.P. \& Tryniecki, J. L. (2007). Maximal eccentric and concentric strength discrepancies between young men and women for dynamic resistance exercise. Journal of Strength \& Conditioning Research, 21(1), 37-40. doi: http://dx.doi.org/10.1519/00124278-200702000-00007

Instituto Nacional de Salud Púbica. (2012). Encuesta Nacional de Salud y Nutrición 2012. Recuperado de http://ensanut.insp.mx/

Krebs, N. F., \& Jacobson, M. S. (2003). Prevention of pediatric overweight and obesity. American Academy of Pediatrics, 112(2), 424-430.Recuperado de http://pediatrics.aappublications.org/content/pediatrics/112/2/424.full.pdf 
Lazaar, N., Aucouturier, J., Ratel, S., Rance, M., Meyer, M., \& Duché, P. (2007). Effect of physical activity intervention on body composition in young children: influence of body mass index status and gender. ActaPediatrica, 96(9), 1321-1325. doi: http://dx.doi.org/10.1111/j.1651-2227.2007.00426.x

López, E. J. M., Peiró, M. R., \& García, J. M. (2011). Grasa corporal mediante bioimpedancia eléctrica en periodo escolar y no escolar. Revista Internacional de Medicina y Ciencias de la Actividad Física y del Deporte, (41), 5-17. Recuperado de https://dialnet.unirioja.es/servlet/articulo?codigo $=3636069$

Marcos-Daccarett, N. J., Núñez-Rocha, G. M., Salinas-Martínez, A. M., Santos-Ayarzagoitia, M., \& Decanini-Arcaute, H. (2007). Obesidad como factor de riesgo para trastornos metabólicos en adolescentes mexicanos, 2005. Revista de Salud Pública, 9(2), 180-193. doi: http://dx.doi.org/10.1590/S0124-00642007000200003

Marsh, A. P., Janssen, J. A., Ambrosius, W. T., Burdette, J. H., Gaukstern, J. E., Morgan, A. R., Nesbit, A.B., Paolini, B., Sheedy, J.L., \& Rejeski, W. J. (2013). The Cooperative Lifestyle Intervention Program-II (CLIP-II): Design and methods. Contemporary clinical trials, 36(2), 382-393.doi:http://dx.doi.org/10.1016/i.cct.2013.08.006

Nakanishi, N., Matsuo, Y., Yoneda, H., Nakamura, K., Suzuki, K., Tatara, K. (2000). Validity of the conventional indirect methods including Friedewald method for determining serum low-density lipoprotein cholesterol level: comparison with the direct homogeneous enzymatic analysis. Journal of Occupational Health, 42(3), 130-137.doi: http://dx.doi.org/10.1539/joh.42.130

Norman, A. C., Drinkard, B., McDuffie, J. R., Ghorbani, S., Yanoff, L. B., \& Yanovski, J. A. (2005). Influence of excess adiposity on exercise fitness and performance in overweight children and adolescents. Pediatrics, 115(6).doi:http://dx.doi.org/10.1542/peds.2004$\underline{1543}$

Organization for Economic Co-operation and Development. (2014). Obesity Update. Recuperado de http://www.oecd.org/health/Obesity-Update-2014.pdf

Rosillo, I., Pituelli, N., Corbera, M., Lioi, S., Miryan, T., D’Arrigo, M.,Gastaldi, L., \& Beloscar, J. (2005). Perfil lipídico en niños y adolescentes de una población escolar. Archivos argentinos de pediatría, 103(4), 293-297. Recuperado de http://www.scielo.org.ar/scielo.php?pid=S032500752005000400003\&script=sci arttext\&t $\underline{\operatorname{lng}=\mathrm{pt}}$

$-14-$

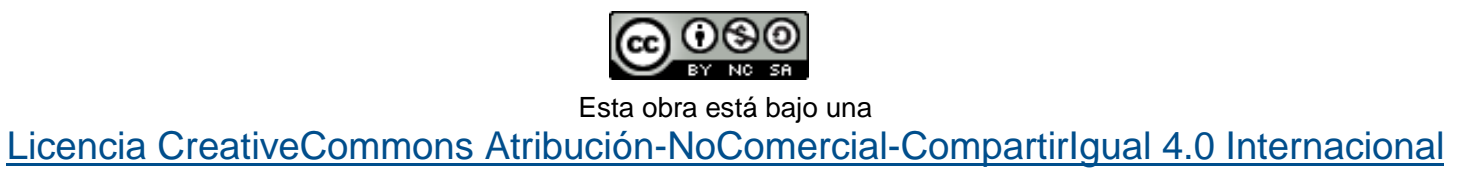


Salazar-Martinez, E., Allen, B., Fernandez-Ortega, C., Torres-Mejia, G., Galal, O., \& LazcanoPonce, E. (2006). Overweight and obesity status among adolescents from Mexico and Egypt. Archives of Medical Research, 37(4), 535-542.doi: http://dx.doi.org/10.1016/i.arcmed.2005.10.014

Sassen, B., Kok, G., Schaalma, H., Kiers, H., \& Vanhees, L. (2010). Cardiovascular risk profile: cross-sectional analysis of motivational determinants, physical fitness and physical activity. BMC Public Health, 10(1), 592. doi: http://dx.doi.org/10.1186/1471-2458-10-592

Stensel, D., Lin, F. P., Ho, T. F., \& Aw, T. C. (2001). Serum lipids, serum insulin, plasma fibrinogen and aerobic capacity in obese and non-obese Singaporean boys. International Journal of Obesity, 25(7), 984-989. doi: http://dx.doi.org/10.1038/sj.ijo.0801667

Stensvold, D., Tjønna, A. E., Skaug, E. A., Aspenes, S., Stølen, T., Wisløff, U., \& Slørdahl, S. A. (2010). Strength training versus aerobic interval training to modify risk factors of metabolic syndrome. Journal of applied physiology, 108(4), 804-810. doi: http://dx.doi.org/10.1152/japplphysiol.00996.2009

Tibana, R. A., da Cunha Nascimento, D., de Sousa, N. M. F., de Souza, V. C., Durigan, J., Vieira, A., Bottaro, M., De Toledo, O., De Almeida, J, Navalta, J.W., Franco, O. L., \& Prestes, J. (2014). Enhancing of Women Functional Status with Metabolic Syndrome by Cardio protective and Anti-Inflammatory Effects of Combined Aerobic and Resistance Training. PlosOne, 9(11). Recuperado de http://journals.plos.org/plosone/article?id=10.1371/journal.pone.0110160

Vaeyens, R., Malina, R. M., Janssens, M., Van Renterghem, B., Bourgois, J., Vrijens, J., \& Philippaerts, R. M. (2006). A multidisciplinary selection model for youth soccer: the Ghent Youth Soccer Project. British Journal of Sports Medicine, 40(11), 928-934. doi: http://dx.doi.org/10.1136/bjsm.2006.029652

Valdespino, J. L., Olaiz, G., Lopez-Barajas, M., Mendoza, L., Palma, O., Velázquez, O., ...\& Sepúlveda, J. (2003). Encuesta Nacional de Salud 2000, Tomo I: Vivienda, población y utilización de servicios de salud. Cuernavaca, México: Instituto Nacional de Salud Pública. Recuperado de http://ensanut.insp.mx/informes/ENSA tomo1.pdf

Villalpando, S., Carrión, C., Barquera, S., Olaiz-Fernández, G., \& Robledo, R. (2007). Body mass index associated with hyperglycemia and alterations of components of metabolic 
syndrome in Mexican adolescents. Salud Pública de México, 49(3), 324-330. doi: http://dx.doi.org/10.1590/S0036-36342007000900003

World Health Organization. (2007). Growth reference data for 5-19 years 2007. Recuperado de http://www.who.int/growthref/en/

Yeung, D., \& Hui, S. (2010). Validity and reliability of skinfold measurement in assessing body fatness of Chinese children. Asia Pacific Journal of Clinical Nutrition, 19(3), 350357.Recuperado

de http://search.informit.com.au/documentSummary;dn=631174250014287;res=IELHEA

Participación: A-Financiamiento, B-Diseñodelestudio, C-Recolección de datos, D-Análisis estadístico e interpretación de resultados, E-Preparacióndel manuscrito.

\section{(c) (7) (-)}

